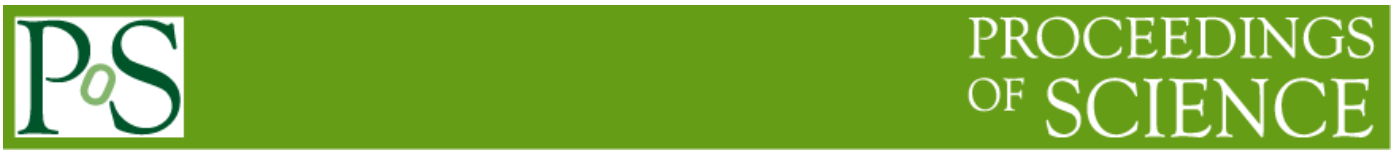

\title{
Time of flight mass measurements of neutron rich nuclides relevant to astrophysical processes.
}

\author{
Alfredo Estrade ${ }^{\star 1,2,3}$, Milan Matoš ${ }^{1,2}$, A. Matthew Amthor ${ }^{1,2,3}$, Daniel Bazin 1 , Ana \\ Becerril $^{1,2,3}$, Thom Elliot ${ }^{1,2,3}$, Alexandra Gade ${ }^{1,3}$, Daniel Galaviz ${ }^{1,2}$, Giuseppe \\ Lorusso $^{1,2,3}$, Jorge Pereira ${ }^{1,2}$, Mauricio Portillo ${ }^{1}$, Andrew Rogers ${ }^{1,2,3}$, Hendrik \\ Schatz ${ }^{1,2,3}$, Andreas Stolz ${ }^{1}$ \\ 1 National Superconducting Cyclotron Laboratory \\ 2 Joint Institute for Nuclear Astrophysics (JINA) \\ 3 Department of Physics and Astronomy \\ Michigan State University, 1 Cyclotron Lab, East Lansing, MI 48824, USA. \\ E-mail: estrade@nscl.msu.edu
}

\section{Dan Shapira}

Oak Ridge National Laboratory, USA.

\section{Ed Smith}

The Ohio State University \& Join Institute of Nuclear Astrophysics, USA.

\section{Mark Wallace}

Los Alamos National Laboratory, USA.

\begin{abstract}
Masses of neutron rich nuclei are of high importance for astrophysics. They are direct inputs for r-process calculations, for modelling nuclear reactions in the crust of accreting neutron stars, etc. For most of the nuclides involved in these processes, the mass has not been determined experimentally. We have recently performed mass measurements by the time of flight technique at the National Superconducting Cyclotron Laboratory at Michigan State University. Details of the experimental technique are discussed, together with the first experiment in the neutron rich Fe region.
\end{abstract}

\footnotetext{
* Speaker
} 


\section{Introduction}

Not only are nuclear masses a fundamental property of the nucleus, but they are deeply linked to our understanding of stellar evolution and nucleosynthesis. Together with observations, a thorough understanding of the nuclear processes involved is necessary to provide quantitative constraints to astrophysical models. The unsatisfactory knowledge of nuclear properties far from stability is one of the main reasons why there still exist important open questions about astrophysical processes involving these nuclides, such as the r-process or nuclear processes in accreting neutron stars $[1,2]$.

The r-process is one of the major nucleosynthesis processes in the universe, responsible for the synthesis of roughly half of the elements heavier than iron. Originally postulated almost fifty years ago, major questions, like its site, are still under debate [1, 3]. Along the r-process path equilibrium between neutron capture and photodisintegration reactions is established along isotopic chains. Since the equilibrium abundances are exponentially sensitive to the neutron separation energy, nuclear masses directly affect the evolution and final abundances produced during the r-process $[4,5]$.

Accreting neutron stars are prime laboratories to study nuclear matter at extreme conditions. Deep inside the crust, the accreted matter can undergo a series of electron captures and pycnonuclear fusion reactions that drive nuclear matter towards the neutron drip line [6, 7]. The energy released by these processes, which is directly related to the nuclear binding energy, will affect the composition and thermal profile of the crust. In transient systems, this can contribute to the observed quiescent luminosity (when accretion shuts off). The heat generated is also important to set the ignition conditions for X-ray bursts and superbursts, which are thermonuclear explosion of the accreted material that take place in the atmosphere and upper crust of the star [8].

The measurement of masses of neutron rich nuclides presents an experimental challenge. Not only do they have small production rates, but their short half lives limit the methods that can be used to determine their masses. As a result, only a handful of nuclides relevant to the aforementioned processes has been directly measured, and current astrophysical calculations have to rely on theoretical predictions. However, the results of different mass models diverge for very neutron rich nuclides with disagreements up to a few $\mathrm{MeV}$, and new measurements are the best tool to test these models [9].

Time-of-flight (TOF) mass measurements are based on the equation of motion of a charged particle in a magnetic field:

$$
B \rho=\frac{m_{0}}{q}\left(\frac{d z}{d t}\right)
$$

where $B \rho$ is the magnetic rigidity of a particle, $q$ its charge, $m_{0}$ its rest mass, $\gamma$ the Lorentz factor, and $d z / d t$ its velocity. The mass is obtained by measuring the time of flight through the beamline relative to nuclides of known mass. The momentum acceptance of the beamline is usually of the order of a few percent. Therefore, the spread in $B \rho$ has to be corrected either with a particle by particle $B \rho$ measurement or by constraining some parameter of the optics setting 
(for example an isochronous tune) [10]. The TOF technique is used in fast radioactive beam facilities where the short time of flight through the beamline system, generally less than $1 \mu \mathrm{s}$, allows for the measurement of very short lived isotopes. Combined with production rates of neutron rich nuclides at modern facilities, such as the NSCL, TOF is one of the techniques that provide mass measurements farther from stability. A review of different methods and recent progress in mass measurements is presented in [11].

Mass measurements of neutron rich nuclides with the TOF technique have been performed at other radioactive beam laboratories $[12,13]$, resulting in statistical uncertainties of the order of $200 \mathrm{keV}$. A first experiment at the National Superconducting Cyclotron Laboratory (NSCL) in Michigan State University was recently completed around ${ }^{68} \mathrm{Fe}$, in the region of $19<\mathrm{Z}<32$.

\section{TOF mass measurements at the NSCL}

At the experiment performed at the NSCL we produced a radioactive ion beam in the region of ${ }^{68} \mathrm{Fe}$ by fragmentation of a relativistic $100 \mathrm{MeV} /$ nucleon ${ }^{86} \mathrm{Kr}$ primary beam in a solid

${ }^{9}$ Be target. Two different production targets with thicknesses of $46 \mathrm{mg} / \mathrm{cm}^{2}$ and $94 \mathrm{mg} / \mathrm{cm}^{2}$ were used to populate a wider region of the chart of the nuclides in the secondary beam without the need to rescale any magnetic fields. This provides a large number of nuclides of very well known mass, which are used for calibration.

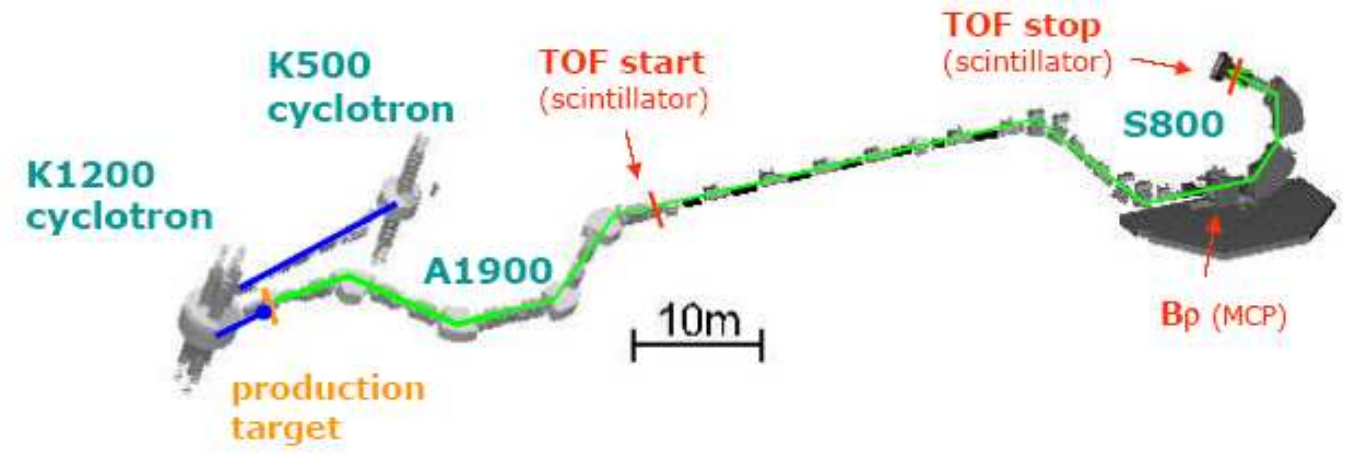

Figure 1: Experimental setup for TOF-B $\rho$ mass measurement experiments at the National Superconducting Cyclotron Laboratory (NSCL).

The time of flight from the focal plane of the A1900 fragment separator to the focal plane of the S800 spectrometer was measured using fast plastic scintillation detectors. This results in a path length of $58 \mathrm{~m}$, which translates into a typical time of flight of $500 \mathrm{~ns}$. The amplitude of the signal for each event was also measured. The S800 spectrograph was operated in dispersion matched optics. In this mode, the beam is dispersed in the vertical position at the S800 dispersive plane (11 cm per percent momentum deviation). Two position sensitive microchannel plate detectors were used at this position ("MCP" in Figure 1) to measure the relative magnetic rigidity and provide tracking of the beam particles. The ion chamber and CRDC detectors at the S800 focal plane were used in order to identify each particle by energy loss and track its position and angle, respectively [14].

In our experimental set up, the timing resolution represents the major contribution to the loss in mass resolving power. For the characteristic time of flight of the particles, a timing 
resolution of the order of $100 \mathrm{ps}$ is required. Since the optical tune of the beamlines is not isochronous from the TOF start to the TOF stop location, the time of flight for each particle has to be corrected by its momentum dependence to attain the desired resolution. Therefore a good position resolution $(<1 \mathrm{~mm})$ is required of the micro channel plate detector located at the $S 800$ dispersive plane. The high degree of momentum dispersion there allows us to precisely correlate particle momentum with position.

\section{Detectors Resolution}

\subsection{Timing Detectors}

Timing detectors made with fast scintillating materials and photomultiplier tubes (PMTs) can provide very good timing characteristics with large detection efficiency, and the ability to sustain high count rates. In order to minimize the spread of the light signal as it travels to the PMTs, we used a small rectangular piece $(1.5$ by $2.54 \mathrm{~cm})$ of BC418 plastic as the scintillating material. The edges were roughened to avoid light reflections inside the scintillator, and thus further reduce the signal spread in time. To minimize beam straggling, and the consequent disruption of the beam's phase space, the thickness of the scintillator was only $0.254 \mathrm{~mm}$. The light signals were collected by two Hamamatsu R4998 PMTs attached to each side of the scintillator. The short rise time of these PMTs $(0.7 \mathrm{~ns})$ resulted in a signal with fast rise time (less than $1 \mathrm{~ns}$ ).

The timing detectors were tested with a $140 \mathrm{MeV} /$ nucleon ${ }^{136} \mathrm{Xe}$ beam in a previous experiment. The resolution achieved by measuring the time signal of one PMT with respect to the other was $\sigma=30 \mathrm{ps}$, after correcting for the particle's position at the plastic scintillator. The test also confirmed that the detectors were able to sustain count rates above $1 \mathrm{MHz}$. The observed resolution is comparable to that of timing scintillators of similar design described elsewhere [15].
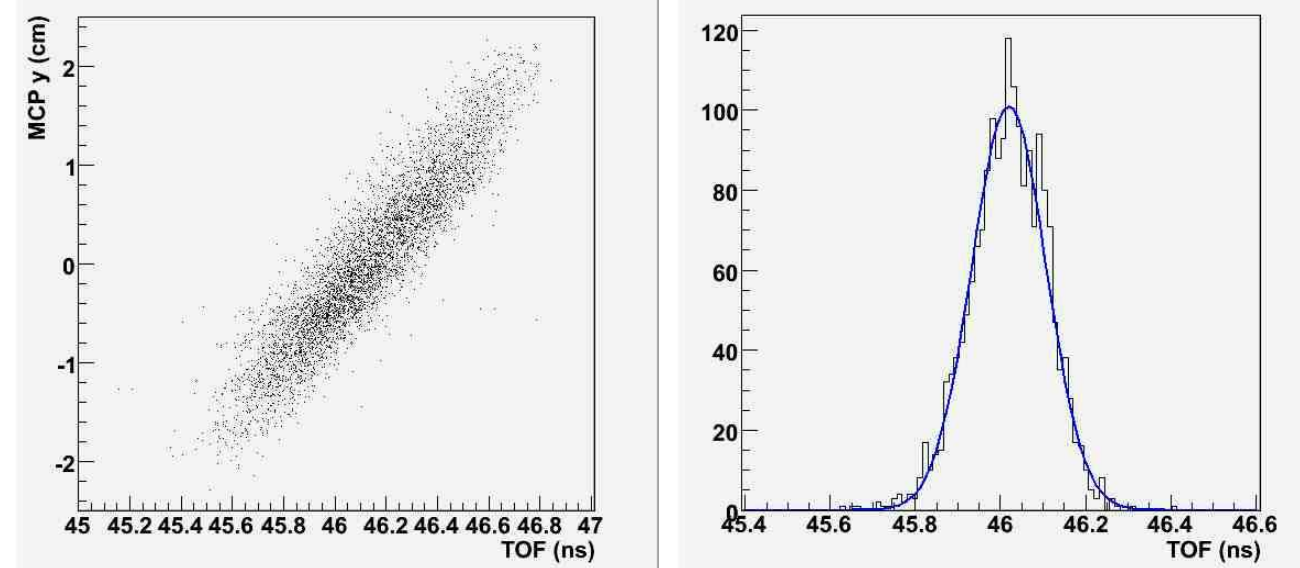

Figure 2: Time of flight measured for ${ }^{79} \mathrm{Ga}$ fragments. The left panel shows the dependence of the time of flight on the magnetic rigidity of the particles, as measured at the S800 dispersive plane. The right panel shows a Gaussian fit to the data with $\sigma=85 \mathrm{ps}$. 
Momentum dependent effects on the time of flight can be corrected for by reconstructing the path of each particle from the position tracking at the S800 dispersive plane and focal plane. Preliminary correction schemes have thus far demonstrated that a time resolution of about $\sigma=$ 85 ps can be obtained (Figure 2).

\subsection{Position Sensitive detectors}

Microchannel plates (MCPs) are position sensitive detectors based on the detection of secondary electrons emitted from a foil in the path of the beam. The secondary electrons are guided by a combination of electric and magnetic fields to a position sensitive microchannel plate where the signal is amplified to generate the position information [16]. They allow position measurement with a minimum material in the path of the beam. For this experiment we used thin $70 \mu \mathrm{g} / \mathrm{cm}^{2}$ Au coated mylar foils.

The position calibration is obtained by sending a defocused beam through a circular hole mask. The data is compared to a Monte Carlo simulation, which studied how the spectrum observed from a single mask hole depends on the intrinsic detector resolution. Results show that we obtained a position resolution of $\sigma=0.3 \mathrm{~mm}$.
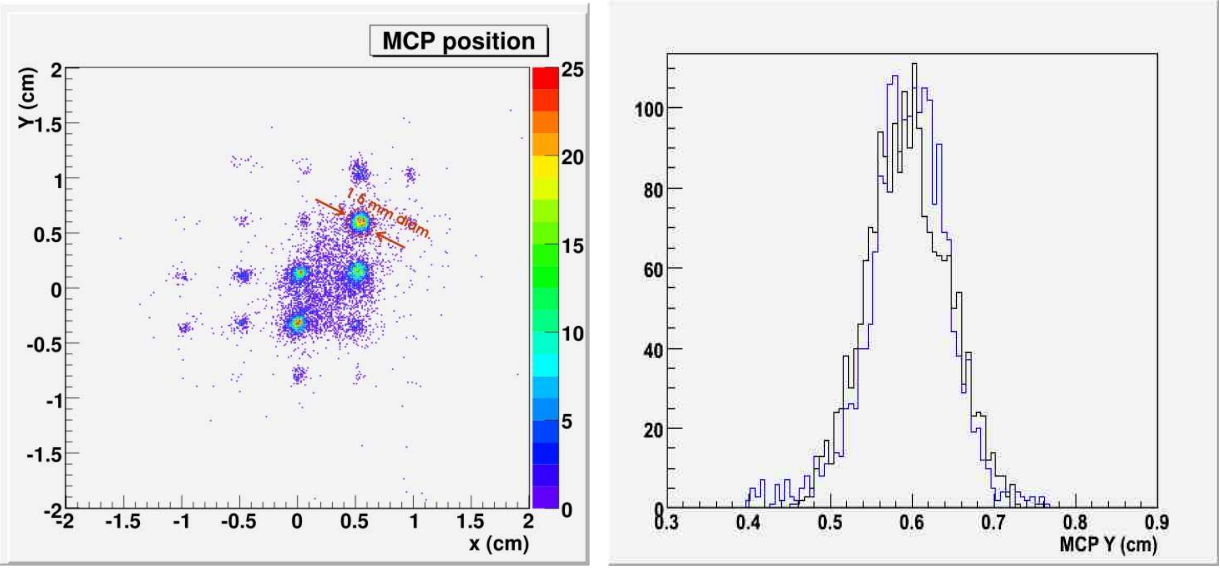

Figure 3: Position calibration of the MCP detector obtained with a mask placed at the MCP foil. The right panel shows the projection on the $\mathrm{y}$-axis of the mask hole at $\mathrm{x}=0.5, \mathrm{y}=0.5$ (blue), and the data from a Monte Carlo simulation for a detector resolution of $\sigma=0.3 \mathrm{~cm}$ (black).

\section{Conclusion}

We have successfully completed a time of flight mass measurement experiment in the region of neutron rich Fe nuclides at the National Superconducting Cyclotron Laboratory (NSCL). Preliminary analysis shows that the performance of the tracking detectors will allow us to take full advantage of the S800 spectrometer resolution, and correct for chromatic effects in the beam. With these corrections, a timing resolution below 100 ps has been achieved. Further analysis may lead to more enhanced correction techniques, such as accounting for the particles interaction with materials in the beamline, in order to provide final mass values. 


\section{References}

[1] K.-L. Kratz et al., ApJ 403 (1993) 216.

[2] H. Schatz, Nucl. Phys. A 746 (2004) 347c.

[3] J. J. Cowan, F.-K. Thielemann, and J. W. Truran, Phys. Rep. 208 (1991) 267.

[4] H. Schatz et al., ApJ 579 (2002) 626.

[5] S. Wanajo et al., ApJ 606 (2004) 1057.

[6] P. Haensel, and J. L. Zdunik, A\&A 404 (2003) L33.

[7] E. F. Brown, ApJ 531 (2000) 988.

[8] E. Brown et al., in these proceedings.

[9] D. Lunney, J. M. Pearson, C. Thibault, Rev. Mod. Phys. 75 (2003) 1021.

[10] M. Matos, in AIP Conference Proceedings 819 (2006) 164.

[11] D. Lunney, EPJ A 25 (2005) 3.

[12] H. Savajols, Hyperfine Interact. 132 (2001) 245.

[13] J. M. Wouters et al., NIM B 26 (1987) 286.

[14] D. Bazin et al., NIM B 204 (2003) 629.

[15] S. Nishimura et al., NIM A 510 (2003) 377.

[16] D. Shapira, T. A. Lewis, and L. D. Hulett, NIM A 454 (2000) 409. 